\title{
Rescue of neurological deficits in a mouse model for Angelman Syndrome by reduction of $\alpha \mathrm{CaMKII}$ inhibitory phosphorylation
}

Geeske M van Woerden ${ }^{1}$, Karen D Harris ${ }^{2}$, Mohammad Reza Hojjati ${ }^{1}$, Richard M Gustin ${ }^{3}$, Shenfeng Qiu ${ }^{2}$, Rogerio de Avila Freire ${ }^{1}$, Yong-hui Jiang ${ }^{4}$, Ype Elgersma ${ }^{1}$, Edwin J Weeber ${ }^{2,3}$

${ }^{1}$ Erasmus MC, University Medical Centre, Department of Neuroscience, PO Box 2040, 3000 CA Rotterdam, The Netherlands

${ }^{2}$ Department of Molecular Physiology and Biophysics, ${ }^{3}$ Department of Pharmacology, Vanderbilt University, Nashville, Tennessee 37235, USA

${ }^{4}$ Department of Molecular and Human Genetics, Baylor College of Medicine, Houston, Texas 770301, USA

Correspondence should be addressed to Y.E. (y.elgersma@erasmusmc.nl) 
Angelman Syndrome (AS) is a severe neurological disorder characterized by mental retardation, motor dysfunction and epilepsy. We now show that the molecular and cellular deficits of an AS mouse model can be rescued by introducing an additional mutation at the inhibitory phosphorylation site of $\alpha$ CaMKII. Moreover, these double mutants do no longer show the behavioral deficits seen in AS mice, suggesting that these deficits are the direct result of increased $\alpha$ CaMKII inhibitory phosphorylation.

Angelman Syndrome (AS) is caused by loss of function of imprinted genes on human chromosome $15 \mathrm{q} 11-13$ or by mutations in the $U B E 3 A$ gene which resides in this region ${ }^{1-4}$. Imprinting of this gene results in exclusive maternal expression in hippocampal neurons and cerebellar Purkinje cells ${ }^{5}$. The $U B E 3 A$ gene encodes E6-AP, a ubiquitin protein ligase, but its role remains elusive. Heterozygous mice with a maternally inherited Ube3a mutation (from here on designated as AS mice) exhibit seizures, motor and cognitive abnormalities similar to the symptoms seen in AS patients ${ }^{6}$. Biochemical analysis of these mice indicates a reduction of calcium/calmodulin-dependent kinase type 2 (CaMKII) activity, and increased phosphorylation of the inhibitory Thr305 and Thr306 site ${ }^{7}$. Because increased CaMKII inhibitory phosphorylation has a severe impact on neuronal function ${ }^{8-10}$, we now investigated whether the increased inhibitory CaMKII phosphorylation is directly responsible for the major deficits seen in AS. Female AS mice were crossed with heterozygous males that carry the targeted $\alpha C a M K I I-T 305 V / T 306 A$ mutation, which prevents $\alpha$ CaMKII inhibitory phosphorylation (designated as CaMKII- $305 / 6^{+/-}$mice) (Supplementary methods). This resulted in offspring with four different genotypes: wild-type (WT) mice, mutants carrying 
the AS mutation or $\alpha \mathrm{CaMKII-} 305 / 6^{+/-}$mutation, and mice carrying the double AS/CaMKII$305 / 6^{+/-}$mutation. As shown previously ${ }^{7}$, Western blot analysis of hippocampal lysates, showed a significant increase of Thr305 phosphorylation in AS mice (130\%; $P<0.05$ Fisher's PLSD). In contrast, inhibitory phosphorylation was significantly reduced in CaMKII-305/6 $6^{+-}$ mutants $(40 \%, P<0.001)$, and remained at this low level in AS/CaMKII-305/6 $6^{+/-}$double mutants (Fig. 1a and Supplementary Fig.1). Moreover, the decreased kinase activity of the AS mutants was restored to near wild-type levels in the AS/CaMKII-305/6 ${ }^{+/-}$double mutants (Fig. 1b). Thus, these mutants are suitable to test to what extend increased CaMKII inhibitory phosphorylation is underlying the neurological phenotype of AS mice.

All mutants appeared healthy, but adult ( $>2$ months) AS mice showed a small (20\%) but significant increase in bodyweight ( $P<0.005$ Fisher's PLSD; Supplementary Fig. 2). Although obesity is not a characteristic feature of the disease, it has been reported in $15 \%$ of all AS children ${ }^{11}$ and in the majority of AS children carrying the Ube $3 a$ mutation ${ }^{12}$. Moreover, obesity is common feature of children with a previously unrecognized form of $\mathrm{AS}^{13}$. Notably, increased bodyweight was absent in AS/CaMKII-305/6 ${ }^{+/-}$double mutants $(P>0.3)$. Since $\alpha$ CaMKII expression is restricted to neurons, these results suggest that the increased bodyweight of AS mice has a neurological basis, and is caused by increased aCaMKII Thr ${ }^{305 / 306}$ phosphorylation.

Epilepsy is a common feature of $\mathrm{AS}^{11,14}$. However, since epilepsy is absent in some AS patients and background-dependent in AS mice ${ }^{8}$, several genes seem to be involved in the development of this phenotype. Nevertheless, since reduced $\alpha$ CaMKII activity can result in epilepsy $^{8}$, it could potentially contribute to the increased seizure propensity of AS mice. Using audiogenic stimulation, we observed seizures in $50 \%$ of the AS mice, whereas no seizures were observed in WT mice or CaMKII-305/6 $6^{+-}$mutants. Notably, there was a 3 fold 
reduction of seizure propensity in AS/CaMKII-305/6 $6^{+/-}$double mutants as compared to the AS mutants $(P<0.05$ Pearson's test, Fig. 1c $)$. This suggests that the increased CaMKII inhibitory phosphorylation strongly contributes to the increased seizure susceptibility of the AS mutants.

Motor coordination deficits are common in all AS patients and AS mice, ${ }^{6,14}$ The $U B E 3 A$ gene is imprinted in cerebellar Purkinje cells ${ }^{5}$ and we have previously shown that $\alpha \mathrm{CaMKII}$ is essential for cerebellar motor learning and Purkinje cell plasticity ${ }^{15}$. Therefore, these deficits could well be caused by deregulation of cerebellar CaMKII. Motor performance of the mutants was assessed using an accelerating rotarod. Indeed there was a significant effect of genotype $\left(\mathrm{F}_{3,31}=6.9, P<0.005\right.$ repeated measures ANOVA): AS mice stayed significantly shorter on the rotarod compared to their wild-type littermates $(P<0.0001$ Fisher's PLSD; Fig.1d). In contrast, CaMKII-305/6 $6^{+-}$mutants performed better than wildtype mice $(P<0.005)$ whereas AS/CaMKII-305/6 $6^{+/}$mice were normal (WT versus AS/CaMKII-305/6 $\left.{ }^{+/-}: P=0.5\right)$. These results suggest that regulation of CaMKII inhibitory phosphorylation plays a critical role in motor coordination performance and that the increased CaMKII inhibitory phosphorylation directly underlies the motor performance deficits of AS mice.

The UBE3A gene is also imprinted in the hippocampus ${ }^{5}$. The critical role of $\alpha$ CaMKII in hippocampus-dependent learning ${ }^{8}$ makes it likely that the increased inhibitory phosphorylation of $\alpha$ CaMKII underlies the cognitive deficits in AS patients and AS mice ${ }^{6}$. Hippocampal-dependent learning was first assessed using the Morris watermaze task. A probe trial given after 6 days of training revealed a significant effect of genotype when the number of target platform crossings was compared between mutants $\left(\mathrm{F}_{3,66}=3.7, P<0.05\right.$ ANOVA). Indeed, whereas WT and CaMKII-305/6 ${ }^{+/-}$mutants showed significant more 
crossings of the target platform position as compared to the other positions, AS mice did not (WT $P<0.0001$; CaMKII-305/6 $6^{+-} P<0.0001$; AS $P=0.16$, Paired t-test between target and average of other positions)(Fig 2a,b). The learning deficit of AS mutants was not due to impaired motor performance or decreased motivation to escape, since all mutants showed similar escape latencies in the hidden platform task $\left(\mathrm{F}_{3,31}=0.8, P=0.5\right.$ repeated measures ANOVA, Fig 2c) as well as in the visible platform task (Supplementary Fig. 3). Moreover, swim speed of AS mice was similar as wild-type mice, and the spatial learning deficits were overcome by additional training (Supplementary Fig. 3). Importantly, the spatial learning deficits of AS mice at day 6 , were again completely rescued by the CaMKII-305/6 $6^{+-}$ mutation, since the AS/CaMKII-305/6 ${ }^{+/-}$double mutants showed a highly selective search strategy (target crossing versus average of other positions $P<0.0001$, Paired t-test).

Hippocampal-dependent learning was also assessed using contextual fear conditioning. Context-dependent memory was tested 24 hours or 7 days following training (Fig 2d). A significant effect of genotype was observed at both time points with AS mice freezing significantly less than any other group ( 24 hours: $\mathrm{F}_{3,25}=9, P<0.0005$ ANOVA all $P<0.01$ Fisher's PLSD; 7 days: $\mathrm{F}_{3,25}=26, P<0.0001$ ANOVA; all $P<0.0001$ Fisher's PLSD). In contrast, CaMKII-305/6 $6^{+/}$mutants showed significantly more freezing in the 7 days memory test (all $P<0.05$ Fisher's PLSD) but not in the 24 hours memory test. Once more, the phenotype was rescued by the AS/CaMKII-305/6 ${ }^{+-}$double mutation (WT versus AS/CaMKII-305/6 $6^{+-} 24$ hours: $P=0.6 ; 7$ days: $P=0.4$ Fisher's PLSD). Since cued conditioning was normal in the AS mice (Supplementary Fig. 4), we conclude that AS mice have a hippocampal learning deficit, and that this deficit can be rescued by decreasing aCaMKII inhibitory phosphorylation. 
$\alpha$ CaMKII inhibitory phosphorylation plays an important role in setting the threshold for the induction of long-term potentiation (LTP): homozygous CaMKII-305/6 mice show increased LTP at sub-threshold stimulation, and normal LTP when a strong stimulation protocol is applied ${ }^{9}$. Conversely, AS mice show a striking LTP deficit, which is rescued by a strong stimulation protocol ${ }^{6,7}$. To directly test whether the LTP deficit of AS mice is due to excessive $\mathrm{Thr}^{305 / 306}$ phosphorylation, we measured LTP in hippocampal slices of the mutants. Consistent with previous findings, AS mice showed a severe LTP deficit compared to wildtype mice $\left(\mathrm{F}_{3,87}=0.3, P<0.0001\right.$ ANOVA; all $P<0.001$ Fishers PLSD)(Fig. 2e). However, LTP was normal in the CaMKII-305/6 $6^{+-}$mice $(P=0.5$ Fishers PLSD) as well as in the AS/CaMKII-305/ $6^{+/-}$double mutants $(P=0.4$ Fishers PLSD), suggesting that the plasticity deficits of AS mice are the result of increased CaMKII inhibitory phosphorylation.

Although further research is needed to link E6-AP to CaMKII inhibitory phosphorylation, this is the first study to report a functional connection between CaMKII and a human learning and memory disorder. It also represents a fundamental breakthrough in our basic understanding of the molecular basis of the motor, seizure and cognitive facets observed in Angelman syndrome.

\section{Acknowledgements}

The authors wish to thank M. Elgersma and M. Aghadavoud Jolfaei for technical support. This work was supported by the Netherlands Organisation for Scientific Research (NWOZonMW), Hersenstichting Nederland (HsN), Netherlands Epilepsy Fund and the Nina Foundation/ Angelman Vereniging. 
1. Knoll, J.H. et al. Am J Med Genet 32, 285-90 (1989).

2. Sutcliffe, J.S. et al. Genome Res 7, 368-77 (1997).

3. Matsuura, T. et al. Nat Genet 15, 74-7 (1997).

4. Kishino, T., Lalande, M. \& Wagstaff, J. Nat Genet 15, 70-3 (1997).

5. Albrecht, U. et al. Nat Genet 17, 75-8 (1997).

6. Jiang, Y.H. et al. Neuron 21, 799-811 (1998).

7. Weeber, E.J. et al. J Neurosci 23, 2634-44 (2003).

8. Elgersma, Y., Sweatt, J.D. \& Giese, K.P. J Neurosci 24, 8410-5 (2004).

9. Elgersma, Y. et al. Neuron 36, 493-505 (2002).

10. Zhang, L. et al. J Neurosci 25, 7697-707 (2005).

11. Smith, A. et al. J Med Genet 33, 107-12 (1996).

12. Moncla, A. et al. J Med Genet 36, 554-60 (1999).

13. Gillessen-Kaesbach, G. et al. Eur J Hum Genet 7, 638-44 (1999).

14. Clayton-Smith, J. Am J Med Genet 46, 12-5 (1993).

15. Hansel, C. et al. Neuron 51, 835-43 (2006). 
Figure 1. Reduction of $\alpha$ CaMKII inhibitory phosphorylation rescues CaMKII activity, seizure propensity and motor performance deficits associated with AS. (a) CaMKII Thr305 phosphorylation is reduced in hippocampal lysates of AS/CaMKII-305/6 ${ }^{+-}$double mutants (b) Restoration of CaMKII activity in hippocampal synaptosomes of AS/CaMKII$305 / 6^{+/-}$double mutants. (c) Propensity to audiogenic seizures of AS mutants is reduced in AS $/$ CaMKII-305/6 $6^{+-}$double mutants. (d) Opposing effects of the AS mutation and CaMKII$305 / 6^{+/-}$mutation on performance on the accelerating rotarod. Asterisks indicate significant differences from wild-type mice. Error bars represent s.e.m. All animal experiments were approved by the Dutch Ethical Committee, or in accordance with Institutional Animal Care and Use Committee guidelines. See text and Supplementary methods for statistics, experimental details and number of subjects used.

Figure 2. Hippocampal learning and plasticity of AS mice is rescued by the CaMKII-305/6 $6^{+-}$ mutation. (a-c) Learning in the Morris watermaze. (a) A probe trial shows that AS mice search less focussed for the platform as compared to WT and CaMKII- $305 / 6^{+/-}$mice. The plots are a visual representation of all search tracks combined, in which the colour indicates the mean time spent at a certain position. The black circle indicates the target platform position used during training. The other (virtual) positions are indicated in white. (b) Quantification of the platform positions as indicated in (a). Bars represent target position, adjacent right, opposite, adjacent left position respectively. Asterisks indicate that target position crossing is significantly more than crossing of the other positions. (c) Escape latency to reach the platform shows no difference between the mutants. (d) AS mutants show impaired contextual conditioning. Freezing was assessed either 24 hours, or 7 days after conditioning. (e) LTP deficits of AS mice are rescued by the CaMKII-305/6 ${ }^{+/-}$mutation. 
Error bars represent s.e.m. See text and Supplementary methods for statistics, experimental details and number of subjects used. 


\section{Supplementary Figure 1}

Decreased inhibitory phosphorylation in hippocampal lysates of AS/CaMKII-305/6 ${ }^{+/}$double mutants. AS mice show a significantly increased CaMKII-T305 phosphorylation, as compared to their WT littermates, which is decreased when crossed with CaMKII-305/6 $6^{+-}$ mice. Western blots were first probed with antibodies directed against Thr305- $\alpha$ CaMKII and after stripping re-probed with $\alpha \mathrm{CaMKII}$ and Actin. To ensure that the staining was within the linear range, the wild-type samples were also loaded at a two fold higher concentration.

\section{Supplementary Figure 2}

The increased bodyweight in AS mice returns to WT level in AS/CaMKII-305/6 ${ }^{+-}$mice. AS mice show a small but significant $(P<0.005)$ increase in bodyweight (females $(2.5$ months old): $\mathrm{F}_{3,48}=5.6, P<0.005$, males (4 months old): $\mathrm{F}_{3,13}=9.6, P<0.005$ ANOVA; Post hoc analysis: all $P<0.005$ Fisher's PLSD), which was absent in AS/CaMKII-305/6 ${ }^{+-}$double mutants ( $P>0.3$ for males and females). Error bars represent s.e.m.

\section{Supplementary Figure 3}

Learning of the hidden platform (a-e) and visible (f) platform watermaze.

AS mice show impaired hippocampal learning at the probe trial given at day 6 compared to their wild type littermates, which is improved after two additional training days. (a) During the probe trial at day 6 AS mice do not spent significantly more time in the target quadrant as compared to all the other quadrants, whereas the AS/CaMKII-305/6 $6^{+-}$mice show high preference for the target quadrant. (b) The learning impairment of the AS mice cannot be ascribed to motor impairments or motivational problems, as they swim as fast as their wild 
type littermates. Only the CaMKII- $305 / 6^{+/}$mice tend to swim faster. $(\mathrm{c}, \mathrm{d}, \mathrm{e})$ At the probe trial given at day 8 , the AS mice caught up with their littermate controls and showed selective searching as judged from the time spend in target quadrant (d) and from the number of

platform crosses (e). To exclude that AS mice have visual or motivational problems that could account for the impaired learning, a visible watermaze was performed and the escape latency was determined. This task shows no effect of genotype $\left(\mathrm{F}_{3,14}=1.4, P=0.3\right.$ repeated measures ANOVA). Error bars represent s.e.m.

\section{Supplementary Figure 4}

AS mice show no deficits in cued conditioning. Cued conditioning test was used to assess whether the contextual conditioning phenotype of the AS mice was hippocampus-dependent. Cued conditioning did not reveal any difference between the different mutants, indicating that the contextual phenotype found in AS mice is indeed hippocampus-dependent $\left(\mathrm{F}_{3,34}\right.$ $=0.6, P=0.6$ repeated measures ANOVA). Error bars represent s.e.m. 


\section{Material and Methods}

\section{Breeding of mutants.}

Mutants with the Ube $3 a$ null mutation (designated as AS mice) and mutants carrying the targeted $\alpha C a M K I I-T 305 V / T 306 A$ mutation which prevents phosphorylation at these residues (designated as CaMKII-305/6 $6^{+/}$mice) were developed as described previously ${ }^{1,2}$. All experiments described in this paper were carried out using hybrid mice in the mixed $129 / \mathrm{Sv}-$ C57BL/6 background. To that end, male $\alpha$ CaMKII-305/6 $6^{+-}$mutants in the C57BL/6 background were crossed with female AS mutants in the 129/Sv background (weight, rotarod, watermaze) or with female AS mutants in the 129/Sv-C57BL/6 background (epilepsy, fear conditioning, LTP).

Mice were genotyped when they were 7-10 days, but the experimenter remained blind for the genotype during data collection and the initial analysis. Genotypes were again established after all experiments were done and the code was then broken to perform the final statistical analysis. All experiments were done with 2-4 month old littermates that were housed in groups of 2-3 per homecage. Genotype groups were approximately sex and age matched. Single-housed mice were excluded for the behavioral studies. The mice were kept on a $12 \mathrm{~h}$ light/dark cycle, with food and water available ad libitum. The behavioral experiments were performed during the light period of the cycle. All animal experiments were approved by the Dutch Ethical Committee, or in accordance with guidelines of Institutional Animal Care and Use Committee of Vanderbilt University.

Western blot analysis of the phosphorylation state of CaMKII.

Lysates were prepared by quickly dissecting the hippocampus followed by homogenization in lysis buffer (10mM TRIS-HCl 6.8, 2.5\% SDS, 2mM EDTA and protease and phosphatase 
inhibitor cocktails (Sigma)). Lysates were then adjusted to $1 \mathrm{mg} / \mathrm{ml}$, and $10 \mu \mathrm{g}$ was used for SDS-PAGE analysis and Western blotting. Western blots were first probed with antibodies directed against Thr305- $\alpha$ CaMKII $\left(\mathrm{pB} 60^{2}, 1: 5000\right)$ and after stripping re-probed with aCaMKII (MAB3119, 1:10000; Chemicon), and Actin (MAB1501R, 1:2000; Chemicon). Blots were quantified using NIH-Image using the $\alpha$ CaMKII signal as loading control, and normalized against wild-type. The number of mice used: WT (4); AS (4); CaMKII-306/5 $5^{+-}$ (4); AS/CaMKII-305/6 ${ }^{+/-}(4)$.

\section{CaMKII Activity Measurement}

Mice were sacrificed by cervical dislocation and hippocampi were quickly isolated in icecold cutting solution and sonicated in cold homogenization buffer containing 4 mM HEPES, $320 \mathrm{mM}$ sucrose and protease inhibitor cocktail (Sigma, Saint Louis, MO). The homogenates were centrifuged at $1500 \mathrm{~g}$ for $10 \mathrm{~min}$. The supernatant was then centrifuged at $16000 \mathrm{~g}$ for 20 min to yield a pellet containing the crude synaptosome fraction. This fraction was resuspended in $200 \mu$ l homogenization buffer to measure the $\mathrm{Ca}^{2+} /$ calmodulin dependent CaMKII activity using the assay kit from Millipore Inc. (14-217). The number of mice used: WT (6); AS (6); CaMKII-306/5 (6); AS/CaMKII-305/6 $^{+/-}$(6).

\section{Weight and seizure susceptibility}

Female AS mice started to show increased bodyweight after 2 months, whereas male AS mice started to become significantly heavier after 4 months. Number of mice used: females (8-9 weeks): WT (13); AS (7); CaMKII-305/6 $6^{+-}$(10); AS/CAMKII-305/6 ${ }^{+/-}$, (13). Males 
(16-18 weeks): WT (4); AS (3); CaMKII-305/6 ${ }^{+/-}$(6); AS/CaMKII-305/6 $6^{+-}$(4). To assess seizure susceptibility we induced audiogenic seizures by vigorously scratching scissors across the metal grating of the mouse cage lid for 20 seconds, or shorter if a seizure developed before that time. The number of mice used in this paradigm: WT (11); AS (10); CaMKII-305/6 ${ }^{+/-}(12) ; \mathrm{AS} / \mathrm{CaMKII-305/6^{+- }}(15)$.

\section{Motor performance.}

We tested motor function in F1 mice using the accelerating rotarod $(4-40 \mathrm{rpm}$, in 5 minutes; model 7650, Ugo Basile Biological Research Apparatus, Varese, Italy). Mice were given two trials per day with a 45-60 min inter trial interval for 4 consecutive days. For each day we calculated the average of the time spent on the rotarod, or of the time until the mouse made 3 consecutive rotations on the rotarod. Maximum duration of a trial was $5 \mathrm{~min}$. The number of mice used: WT (10); AS (7); CaMKII-305/6 $6^{+/-}$(10); AS/CaMKII-305/6 ${ }^{+/-}$(8).

\section{Water Maze.}

To test spatial memory we used the Water Maze. Prior to the test mice were handled extensively ( $2 \mathrm{~min} /$ day; 5 days). Our pool is $1.2 \mathrm{~m}$ in diameter and has an $11 \mathrm{~cm}$ diameter platform submerged $1 \mathrm{~cm}$ below the water surface. The water is painted milk-white with nontoxic paint and water temperature is kept constant at $26^{\circ} \mathrm{C}$. We use dimmed lighting, and mouse-tracking is performed using SMART version 2.0 (Panlab, Barcelona, Spain). Mice were given 2 trials per day, with $30 \mathrm{sec}$ inter trial interval for 8 consecutive days. At a training session, the mouse was first placed on the platform for $30 \mathrm{sec}$. Then it was placed in the water at a pseudo-random start position and it was given a maximum of 60 seconds to find the platform. If the mouse did not find the platform within 60 seconds, it was placed 
back on the platform. After 30 seconds on the platform, this training procedure was repeated once more. The platform position remained at the same position during all trials.

One hour after the training on day 6 and day 8 , a probe trial was given to test spatial learning. Mice were placed on the platform for 30 seconds, after which the platform was removed from the pool and the mice were placed in the pool at the opposite side of the previous platform position. The mice were then allowed to search for the platform for 60 seconds. The number of mice used for this paradigm: WT (18); AS (11); CaMKII-306/5 $5^{+-}$ (18); AS/CaMKII-305/6 $6^{+/-}(14)$.

The visible water maze was performed done in the same way as the hidden platform water maze, except that this time the platform was flagged with a small cue on top the platform. The number of (naïve) mice used for this paradigm: WT (6); AS (5); CaMKII$306 / 5^{+/-}$(4); AS/CaMKII-305/6 ${ }^{+/-}(3)$.

\section{Fear Conditioning.}

Fear conditioning was performed in a testing chamber $(26 \times 22 \times 18 \mathrm{~cm}$; San Diego Instruments, San Diego, CA) made of Plexiglas equipped with a grid floor via which the foot shock could be administered and a CCD camera to monitor activity. The conditioning chamber was placed inside a soundproof isolation cubicle. Training and testing occurred in the presence of white light and white noise. Each mouse was placed inside the conditioning chamber for 150 seconds before the onset of a $2 \mathrm{~s}$ foot shock $(0.5 \mathrm{~mA})$. After 150 seconds, a second foot shock was delivered, and the mouse was returned to its home cage after another 
$2.5 \mathrm{~min}$. Testing of context-dependent fear was performed either 24 hours or 1 week after the conditioning in the same context.

For cued conditioning, each mouse was placed inside the conditioning chamber for 2 min before the onset of a conditioned stimulus (CS; an $85 \mathrm{~dB}$ tone), which lasted for $30 \mathrm{~s}$. training occurred in the presence of white light and white noise. A $2 \mathrm{~s}$ US foot shock $(0.5$ $\mathrm{mA}$ ) was delivered immediately after the termination of the CS. Each mouse remained in the chamber for an additional $120 \mathrm{~s}$, followed by another CS-US pairing. Each mouse was returned to its home cage after another $2.5 \mathrm{~min}$. Cued fear conditioning was tested in the presence of a masked context consisting of a small Plexiglas cube attached to an opaque Plexiglas floor insert, different lighting conditions, and a vanilla odor. Each mouse was placed in this novel context for $3 \mathrm{~min}$ at approximately $24 \mathrm{~h}$ after training, and they were exposed to the CS for another 3 min.

Freezing behavior was recorded and processed by SDI Photobeam Activity System software throughout each testing session. The mouse was considered to be freezing after a lack of movement for $2 \mathrm{~s}$. Number of animals used: 24 hours context: WT (6); AS (6); CaMKII-305/6 ${ }^{+/-}(8)$; AS/CaMKII-305/6 $6^{+-}(9)$; 1 week context: (WT (7); AS (8); CaMKII$305 / 6^{+/-}$(7); AS/CaMKII-305/6 ${ }^{+/-}(8) .24$ hours cued: WT (11); AS (11); CaMKII-305/6 $6^{+/-}$ (7); AS/CaMKII-305/6 $6^{+/-}(9)$;

Shock threshold was assessed by placing the animal in the conditioning chamber and by delivering foot shocks starting at $0.075 \mathrm{~mA}$ and increasing by $0.05 \mathrm{~mA}$ every $30 \mathrm{~s}$. The experiment was terminated at the shock intensity sufficient to induce vocalization. There was no difference in shock sensitivity between the groups $\left(\mathrm{F}_{3,75}=0.53, P=0.9\right.$ ANOVA $)$. 


\section{Hippocampal slice preparation and electrophysiology}

The brain was quickly removed and placed in ice-cold high sucrose cutting solution containing (in mM): 110 sucrose, $60 \mathrm{NaCl}, 3 \mathrm{KCl}, 28 \mathrm{NaHCO}_{3}, 1.25 \mathrm{NaH}_{2} \mathrm{PO}_{4}, 7 \mathrm{MgCl}_{2}, 0.5$ $\mathrm{CaCl}_{2}, 0.6$ sodium ascorbate, and $5 \mathrm{D}$ - glucose, $\mathrm{pH}$ 7.3-7.4. Horizontal $400 \mu \mathrm{m}$ sections were cut in sucrose cutting solution using a Vibratome. The slices were stored in $95 \% \mathrm{O}_{2} / 5 \% \mathrm{CO}_{2}$ equilibrated artificial CSF containing (in $\mathrm{mM}$ ): $125 \mathrm{NaCl}, 2.5 \mathrm{KCl}, 1.25 \mathrm{NaH}_{2} \mathrm{PO}_{4}, 28$ $\mathrm{NaHCO}_{3}, 1.0 \mathrm{MgCl}_{2}, 2.0 \mathrm{CaCl}_{2}$, and $10 \mathrm{D}$ - glucose, (pH 7.3-7.4) and kept at room temperature for at least 1 hour before switching to the interface chamber supported by a nylon mesh and allowed to recover for a minimum of 1 hour prior to recording. The chamber was kept at $32 \pm 0.5^{\circ} \mathrm{C}$ with a laminar ACSF flow rate of $2-3 \mathrm{ml} / \mathrm{min}$. Extracellular field recordings were obtained from area CA1 of the stratum radiatum. Stimulations were supplied with a bipolar Teflon-coated platinum electrode and recordings were obtained with the use of a glass microelectrode field with ACSF with tip diameter about $1 \mu \mathrm{m}$ (1-4 M $\Omega$ electrical resistance). Tetani used to evoke CA1 LTP consisted of two trains of $100 \mathrm{~Hz}$ stimulation for 1s with each train separated by a 20 s interval. Stimulus intensities were adjusted to give pEPSPs (population excitatory postsynaptic potentials) with slopes that were $<50 \%$ of the

maximum determined from an input/output curve. Potentiation was measured as the normalized increase of the mean pEPSP for the duration of the baseline recording. Experimental results were obtained from those slices that exhibited stable baseline synaptic transmission for a minimum of 30 min prior to the delivery of the LTP- inducing stimulus. For recording we used an Axon 1320 Digidata data acquisition hardware operated by Axon 
pClamp 9.2 software. We used maximally two slices per mouse. The number of slices used for the experiment are: WT (11); AS (6); CaMKII-305/6 ${ }^{+/-}$(6); AS/CaMKII-305/6 ${ }^{+/-}$(6).

Statistical analysis.

StatView data analysis software was used for statistical analysis. An ANOVA or repeated measures ANOVA was used for multiple group data, to test for the effect of genotype. If this was significant, the data was further analyzed using the Fisher PLSD Post Hoc test. All figure data represents mean \pm SEM. An analysis with a value of $p<0.05$ was considered to be statistically significant (indicated by an asterisk in the figures).

\section{References}

1. Jiang, Y.H. et al. Mutation of the Angelman ubiquitin ligase in mice causes increased cytoplasmic p53 and deficits of contextual learning and long-term potentiation. Neuron 21, 799-811 (1998).

2. Elgersma, Y. et al. Inhibitory autophosphorylation of CaMKII controls PSD association, plasticity, and learning. Neuron 36, 493-505 (2002). 


\section{Fig. 1 Van Woerden et al.}
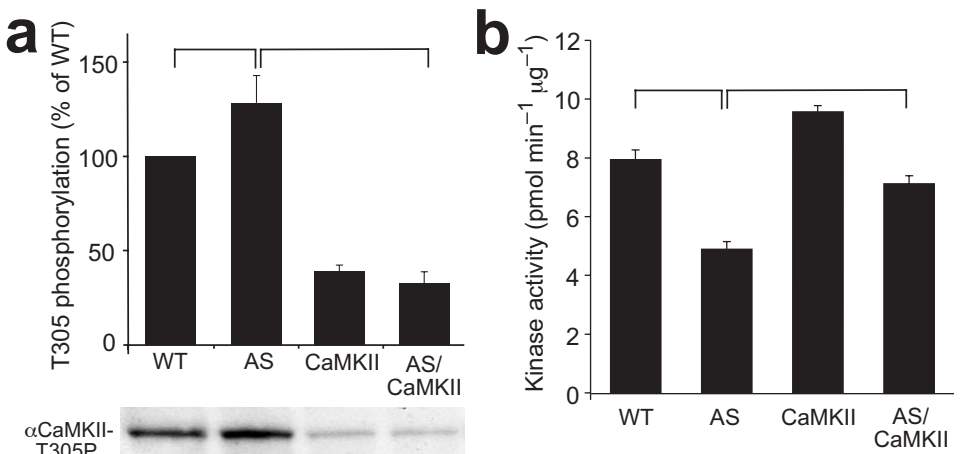

$\alpha \mathrm{CaMKII-}$
T305P
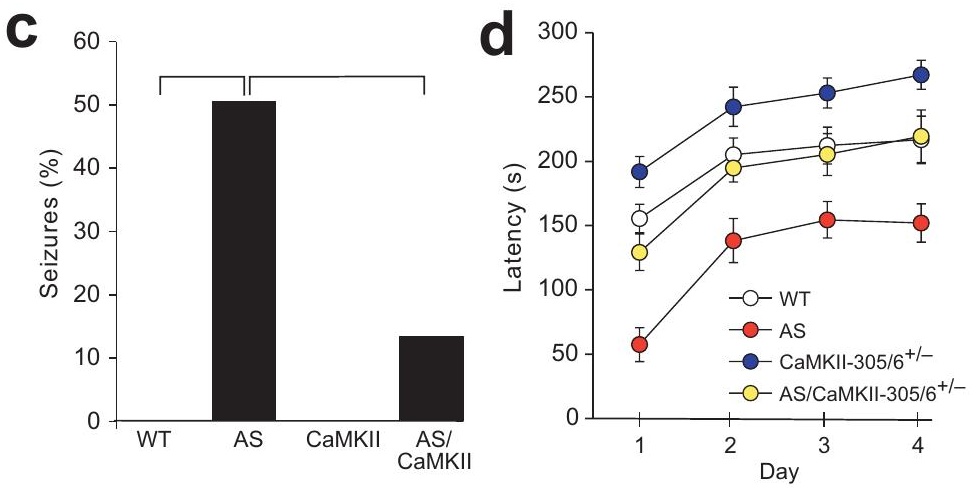
a
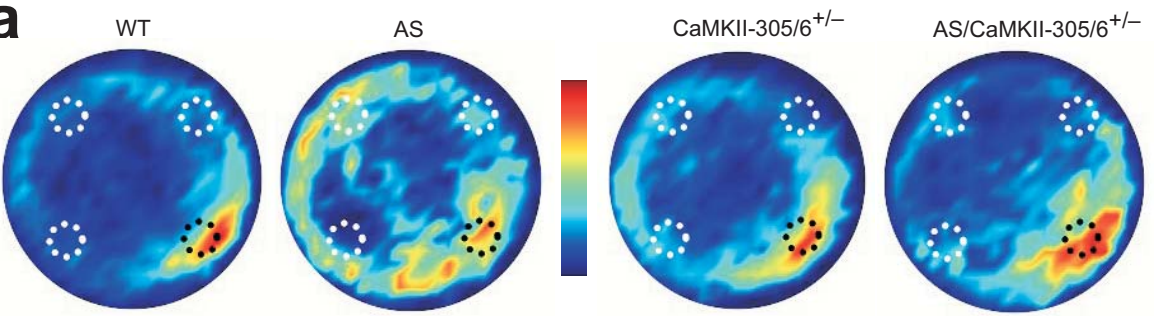

b

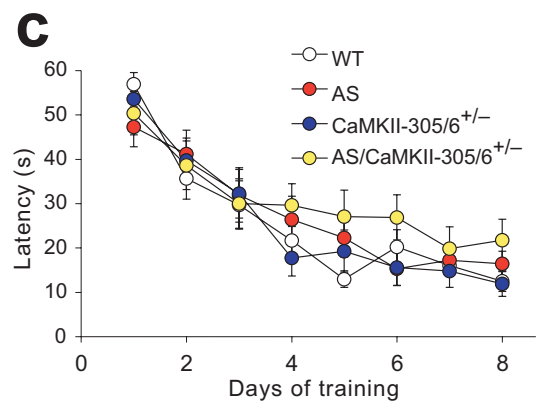

d
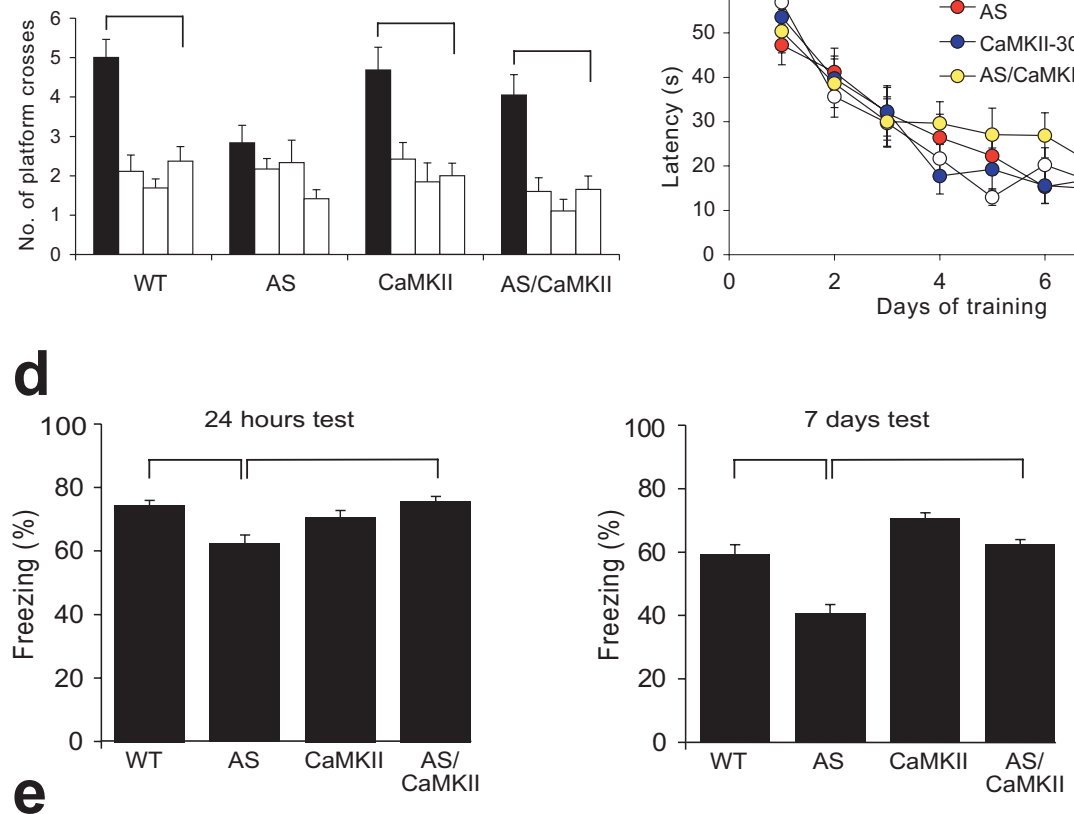

8

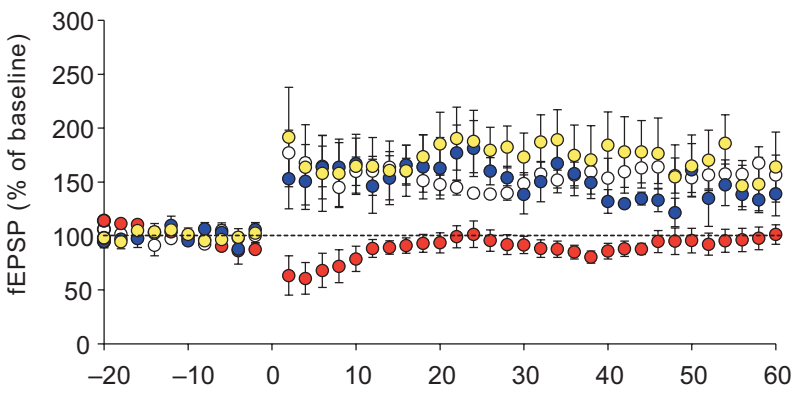

OWT

- AS

- CaMKII-305/6 $6^{+/-}$

o AS/CaMKII-305/6 ${ }^{+/-}$ 


\section{Supplementary Fig. 1 Van Woerden et al.}

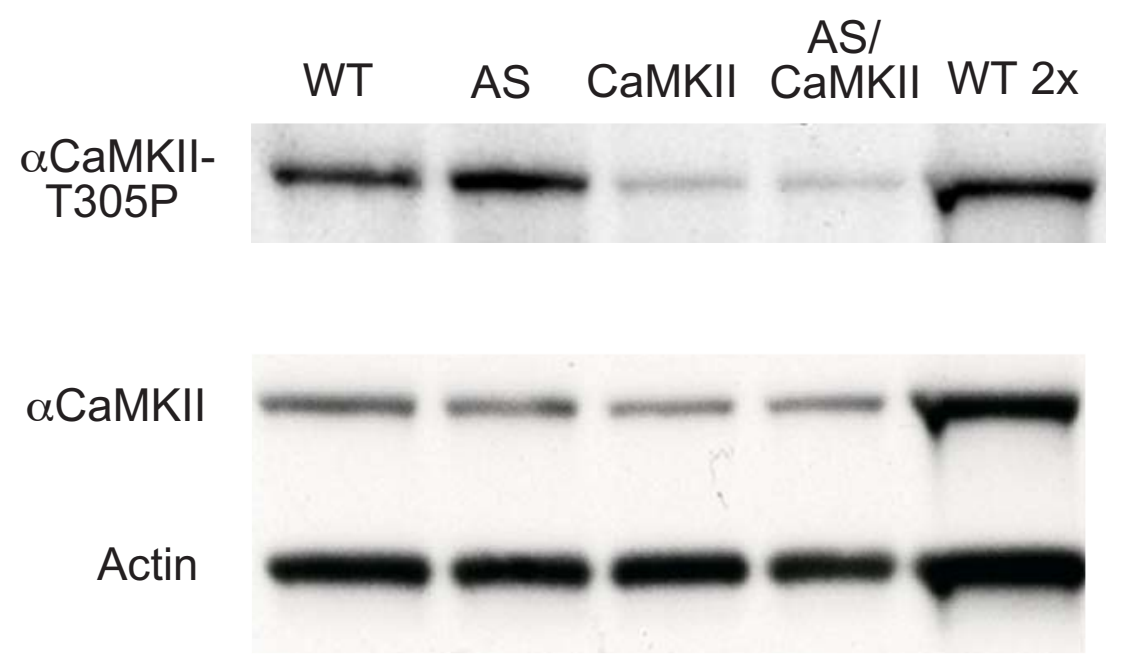

Decreased inhibitory phosphorylation in hippocampal lysates of AS/CaMKII305/6 ${ }^{+-}$double mutants. AS mice show significantly increased CaMKII-T305 phosphorylation, compared to their WT littermates, which is decreased when crossed with CaMKII-305/6 $6^{+-}$mice. Western blots were first probed with antibodies directed against Thr305-CaMKII and after stripping re-probed with CaMKII and Actin. To ensure that the staining was within the linear range, the wild-type samples were also loaded at two fold higher concentration. 


\section{Supplementary Fig. 2 Van Woerden et al.}
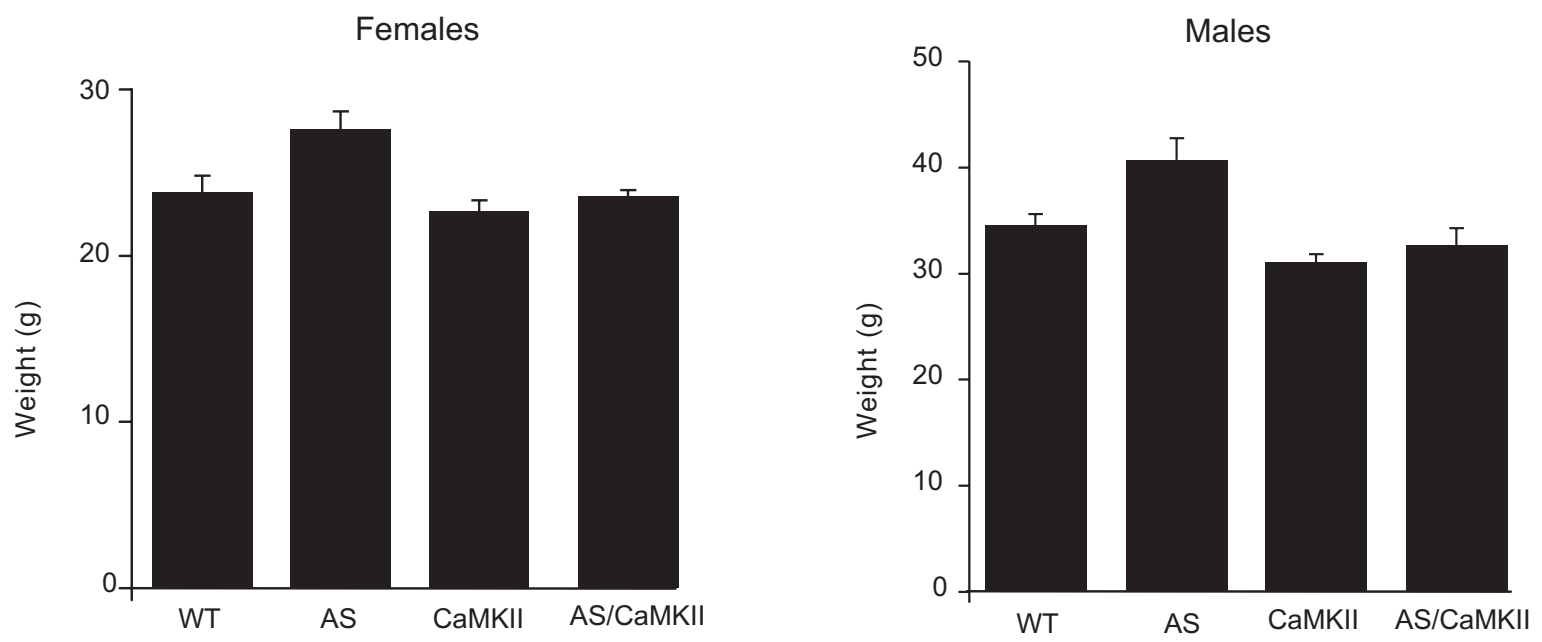

The increased bodyweight in AS mice returns to WT level in AS/CaMKII-305/6 ${ }^{+-}$ mice. AS mice show a small but significant $(P<0.005)$ increase in bodyweight (females (2.5 months old): $F_{3,48}=5.6, P<0.005$, males (4 months old): $F_{3,13}=9.6, P<0.005$ ANOVA; Post hoc analysis: all $P<0.005$ Fisher's PLSD), which was absent in AS/CaMKII-305/6 $6^{+-}$ double mutants ( $P>0.3$ for males and females). Error bars represent SEM. 
Supplementary Fig. 3 Van Woerden et al.
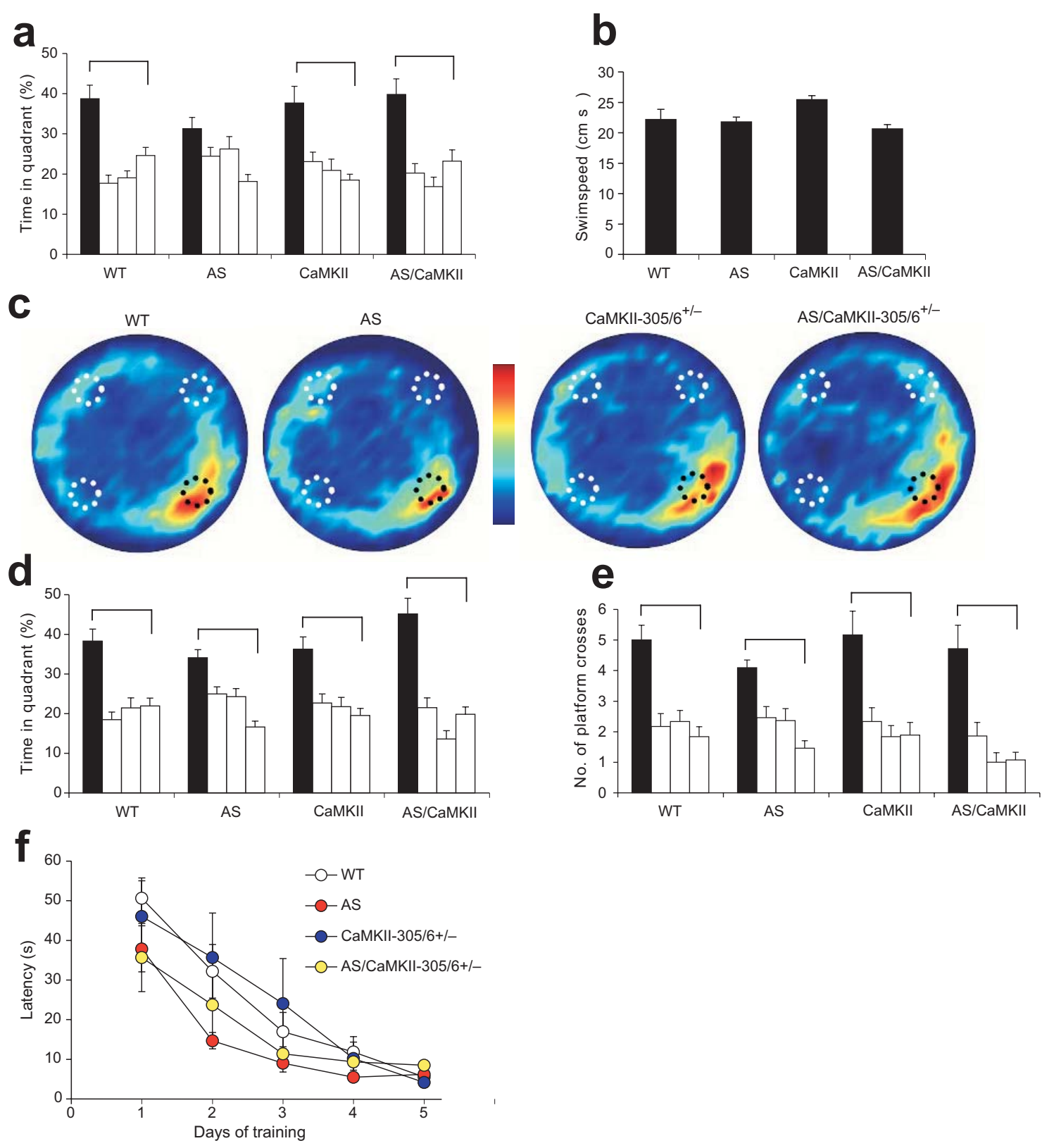

Learning of the hidden platform (a-e) and visible (f) platform watermaze. AS mice show impaired hippocampal learning at the probe trial given at day 6 compared to their wild type littermates, which is improved after two additional training days. (a) During the probe trial at day 6 AS mice do not spent significantly more time in the target quadrant as compared to all the other quadrants, whereas the AS/CaMKII-305/6 ${ }^{+/-}$mice show high preference for the target quadrant. (b) The learning impairment of the AS mice cannot be ascribed to motor impairments or motivational problems, as they swim as fast as their wild type littermates. Only the CaMKII-305/6 ${ }^{+/-}$mice tend to swim faster. (c,d,e) At the probe trial given at day 8 , the AS mice caught up with their littermate controls and showed selective searching as judged from the time spend in target quadrant (d) and from the number of platform crosses (e). To exclude that AS mice have visual or motivational problems that could account for the impaired learning, a visible watermaze was performed and the escape latency was determined. This task shows no effect of genotype $(F 3,14=1.4, P=0.3$ repeated measures ANOVA). Error bars represent SEM. 


\section{Supplementary Fig. 4 Van Woerden et al.}

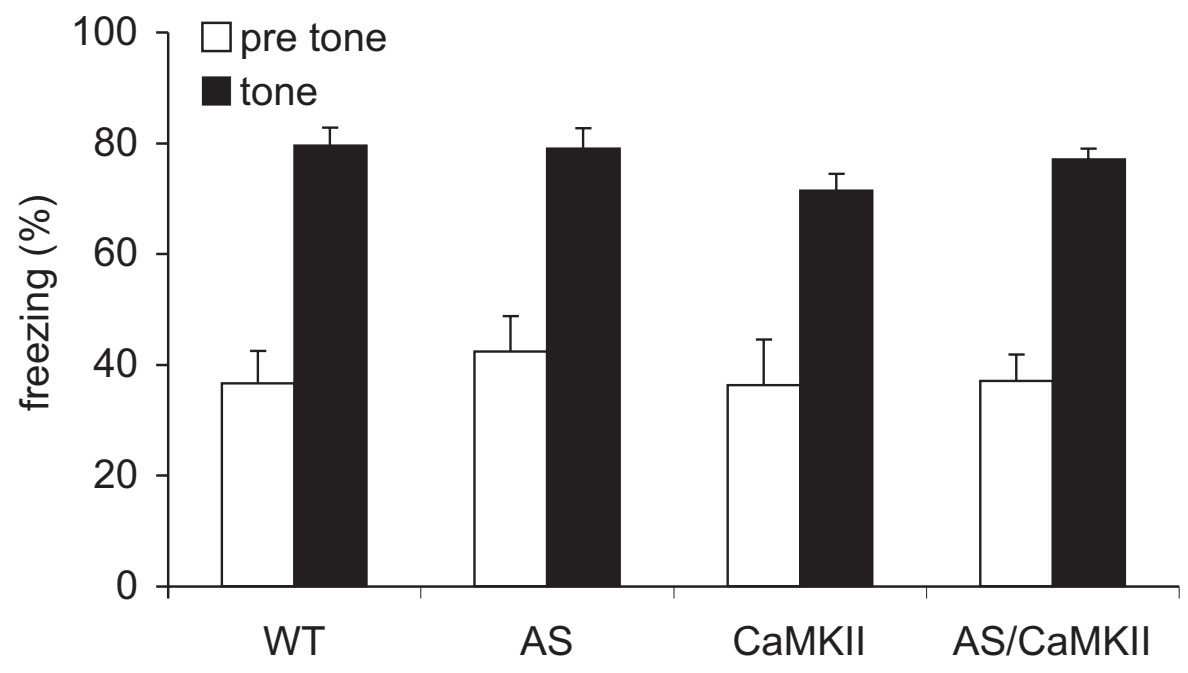

AS mice show no deficits in cued conditioning. Cued conditioning test was used to assess whether the contextual conditioning phenotype of the AS mice was hippocampus-dependent. Cued conditioning did not reveal any difference between the different mutants, indicating that the contextual phenotype found in AS mice is indeed hippocampus-dependent $\left(F_{3,34}=0.6, P=0.6\right.$ repeated measures ANOVA). Error bars represent SEM. 